\title{
Corrosion inhibition of mild steel in nitric acid media by some Schiff bases derived from anisalidine
}

\author{
N. Saxena, S. Kumar, M.K. Sharma, S.P. Mathur* \\ Maharshi Dayanand Saraswati University, Department of Pure and Applied Chemistry, Ajmer, Rajasthan (India) 305009, \\ e-mail: Sudesh_kumar03@rediffmail.com \\ "Corresponding author: sudeshneyol@gmail.com
}

\begin{abstract}
Corrosion inhibition performance of mild steel in nitric acid solution containing different concentration of anisalidine derivative Schiff bases viz. N- (4-nitro phenyl) p-anisalidine (SB1), N- (4-chloro phenyl) p-anisalidine (SB2), $\mathrm{N}$ - (4-phenyl) p-anisalidine (SB3), N- (4-methoxy phenyl) p-anisalidine (SB4), N- (4-hydroxy phenyl) p-anisalidine (SB5) has been investigated using mass loss, thermometric and potentiostate polarization technique. Inhibition efficiencies of Schiff bases have been evaluated at different acid strength. The inhibition efficiency was found larger than their parent amines. Inhibition efficiencies of synthesized Schiff bases increase with inhibitor concentration. Inhibition efficiency increases up to $98.32 \%$ with ansalidine derivative Schiff base.
\end{abstract}

Keywords: Schiff base, degradation, inhibitor and mild steel.

\section{INTRODUCTION}

Mild steel is widely used as an engineering material and its corrosion in acidic medium is of great economic importance. Schiff bases have gained importance over the years because of their use as corrosion inhibition. The exposure can be most severe but in many cases, corrosion inhibitors are widely used in industry to prevent or to reduce corrosion rate of metallic materials in acidic media ${ }^{1,2}$. Schiff bases posses $-\mathrm{C}=\mathrm{N}$ - imine linkage which is supposed to be responsible for their complexion behaviors towards metal ions and corrosion inhibition action $^{3,4}$. Corrosion commonly occurs at metal surfaces in the presence of oxygen and moisture, involving two electrochemical reactions. Oxidation takes place at the anodic site and reduction occurs at the cathodic site. In acidic medium hydrogen evolution reaction predominates. Corrosion inhibitors reduce or prevent these reactions. They are adsorbed on metal surface and form a barrier to oxygen and moisture by complexing with metal ions or by removing corrodants from the environment. Some of the inhibitors facilitate the formation of the passivating film on the metal surface.

Generally, the organic compounds containing hetero atoms like $\mathrm{O}, \mathrm{N}, \mathrm{S}$, and in some cases $\mathrm{Se}$ and $\mathrm{P}$ are found to have the function as very effective corrosion inhibitors ${ }^{5-14}$. The efficiency of these compounds depends upon electron density present around the heteroatoms ${ }^{15}$. Inhibition efficiency also depends upon the number of adsorption active centers in the molecule, their charge density, molecular size, mode of adsorption and formation of metallic complexes. Heteroatoms capable of forming coordinate-covalent bond with metal owing to their free electron pairs. Also the compounds with $\pi$-bonds generally exhibit good inhibitive properties due to an interaction of $\pi$-orbital with their structure which makes them effective potential corrosion inhibitors ${ }^{\mathbf{1 6}}$.

Corrosion of mild steel and its alloys in different acid media has been extensively studied ${ }^{17,18}$. The present research paper deals with the preparation of five Schiff bases viz. N- (4-nitro phenyl) p-anisalidine (SB1), N- (4-chloro phenyl) p-anisalidine (SB2), N- (4-phenyl) p-anisalidine (SB3), N- (4-methoxy phenyl) p-anisalidine (SB4), $\mathrm{N}$ - (4-hydroxy phenyl) p-anisalidine (SB5).

\section{EXPERIMENTAL}

\section{Preparation of Schiff bases}

Schiff bases are prepared by refluxing equimolar quantities of respective carbonyl compounds with amino compounds in minimum quantity of solvent.

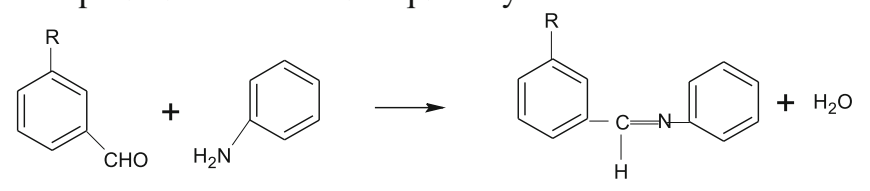

Where $\mathrm{R}=\mathrm{H}$, alkyl or aryl.

\section{Specimen preparation}

Mild steel specimens of the composition of $0.12 \% \mathrm{C}$, $0.32 \% \mathrm{Mn}, 0.05 \% \mathrm{~S}, 0.02 \% \mathrm{SiO}_{2}$ and the rest of $\mathrm{Fe}$ of rectangular specimens dimension $5.0 * 2.5 * 0.02 \mathrm{~cm}^{3}$ containing a small hole of $2 \mathrm{~mm}$ diameter near the upper edge were employed for the determination of corrosion rate in a beaker containing $50 \mathrm{ml}$ of test solution at $23^{\circ} \mathrm{C}$ with a precision of $\pm 0.5^{\circ} \mathrm{C}$ and left exposed to air. While being buffed to produce a mirror finish of mild steel, they were cleaned with emery paper then degreased with acetone. The specimens were suspended by glass hook; the degree of surface coverage $(\theta)$ can be calculated as ${ }^{19}$

$\theta=\frac{(\Delta \mathrm{Mu}-\Delta \mathrm{Mi})}{\Delta \mathrm{Mu}}$

Where $\theta$ surface coverage and $\Delta \mathrm{Mu}$ and $\Delta \mathrm{Mi}$ are the mass loss of the metal in uninhibited and inhibited acid. The corrosion rate mmpy (milimeter penetration per year) can be obtained by the following equation:

$(543 \times \mathrm{W})$

Corrosion rate $($ mmpy $)=\frac{(\text { Area } \times \text { Time } \times \text { Metal density })}{(\text { Tis }}$

Where mass loss is expressed in $\mathrm{mg}$, the area is expressed in $\mathrm{cm}^{2}$ of the metal surface exposed, time is expressed in the hours of exposure \& metal density is expressed in $\mathrm{gm} / \mathrm{cm}^{3}{ }^{20}$.

The inhibition efficiency was also determined using a thermometric technique ${ }^{21}$. These specimens $5.0 * 2.5 * 0.02 \mathrm{~cm}^{3}$ stagnant in a glass hook and immersed in a beaker containing $50 \mathrm{ml}$ of the test solution at $23^{\circ} \mathrm{C}$ 
with a precision of $\pm 0.5^{\circ} \mathrm{C}$ were left exposed to air. Evaporation losses were made up with deionized water. After the test the specimens were cleaned with benzene. The duplicate experiments were performed in each case and the mean values of the mass loss were calculated.

\section{Test solution preparation}

The acidic solution was prepared by using de-ionized water. All the chemicals used were of analytical reagent quality. The temperature changes were measured at an interval of one minute using a thermometer with a precision of $\pm 0.5^{\circ} \mathrm{C}$. The temperatures increased slowly at first then rapidly and attained a maximum temperature. The recorded percentage inhibition efficiencies were calculated as:

$\eta=\frac{\left(\mathrm{RN}_{\mathrm{f}}-\mathrm{RN}_{\mathrm{i}}\right)}{\mathrm{RN}_{\mathrm{f}}} \times 100$

Where $R N_{i}$ and $R_{f}$ are the reaction number in the presence and absence of inhibitors respectively and RN $\left({ }^{\circ} \mathrm{C} / \mathrm{min}\right)$ is defined $\mathrm{as}^{22}$ :

$R N=\frac{\left(T_{m}-T_{0}\right)}{t}$

Where $T_{m}$ and $T_{o}$ are the maximum and initial temperature, respectively and $t$ is the time required to reach the maximum temperature.

\section{Polarization techniques}

The experimental set up used for the polarization measurements include a three-electrode cell and potentiostat (Elico Model CL-95) with a sweep generator was used. The saturated calomel electrode was used as a reference electrode. The variation of the mild steel electrode potential under open circuit conditions in the test solution were determined. Spontaneous reaction takes place on the metal surface due to a potential difference between cathodes and anodes when metal as an electrode is immersed in the aqueous environment, due to activation polarization both reaction rates decrease. The potential and corresponding current densities are called as corrosion potential (Ecorr) and corrosion density $\left(I_{\text {corr }}\right)$ respectively. Cathodic and anodic polarization of the working electrode and the curves shifts towards lower current densities with increasing the concentration of additives ${ }^{23}$.

$I_{\text {corr }}=\frac{\{\beta a \beta c\}}{2.303(\beta a+\beta c)} \times \frac{1}{R p}=\left\{\frac{\Delta i}{\Delta E}\right\}_{\text {Ecorr }}$

The values of $\beta a$ and $\beta c$ and $R p, I_{\text {corr }}$ can be calculated using the above equation.

The percentage inhibition efficiency $(\eta \%)$ can be calculated as follows:

$\eta \%=\frac{I-I_{0}}{I_{0}} \times 100$

Where $I$ and $I_{0}$ are the corrosion currents in uninhibited and inhibited solution espectively.

The corrosion rate can be calculated from the $I_{\text {corr }}$ values using the relation:

$C R($ mmpy $)=\frac{0.1288 \times I_{\text {corr }} \times E}{D}$

Current density $=\frac{\text { Current }}{\text { Area }}$
Where $I_{\text {corr }}$ is the corrosion current in $\mu \mathrm{A} / \mathrm{cm}^{2}, \mathrm{E}$ is the equivalent mass of the metal in $\mathrm{mg}$ and $\mathrm{D}$ is the density in $\mathrm{gm} / \mathrm{cm}^{324}$.

The corrosion rate was converted from mpy to mmpy using the following relation

$1 \mathrm{mpy}=0.0254 \mathrm{mmpy}$

\section{RESULTS AND DISCUSSION}

The inhibition efficiency (\%) calculated from the mass loss measurement for nitric acid media and inhibitors are given in Tables (1-4). It is observed that the inhibition efficiency increases with an increase in the concentration of the inhibitor (Fig. 1-4). The corrosion rate decreases with increases in the concentration of the inhibitors. Table 1 shows the maximum $93.43 \%$ inhibition efficiency for mild steel in $0.1 \mathrm{~N}$ nitric acid solution whereas $0.5 \mathrm{~N}$ shows $94.79 \%$, respectively (Table 2). The Fig.1 and 2 confirm above-mentioned results.

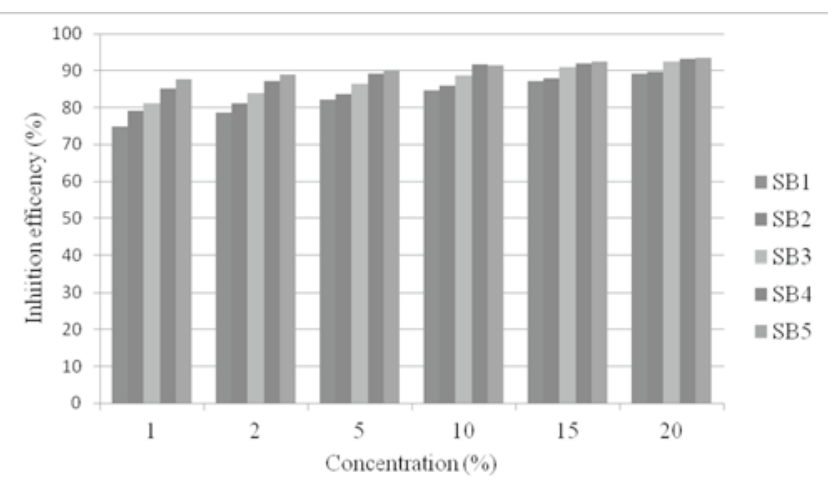

Figure 1. Inhibition efficiency v/s concentration of mild steel in $0.1 \mathrm{~N} \mathrm{HNO}_{3}$

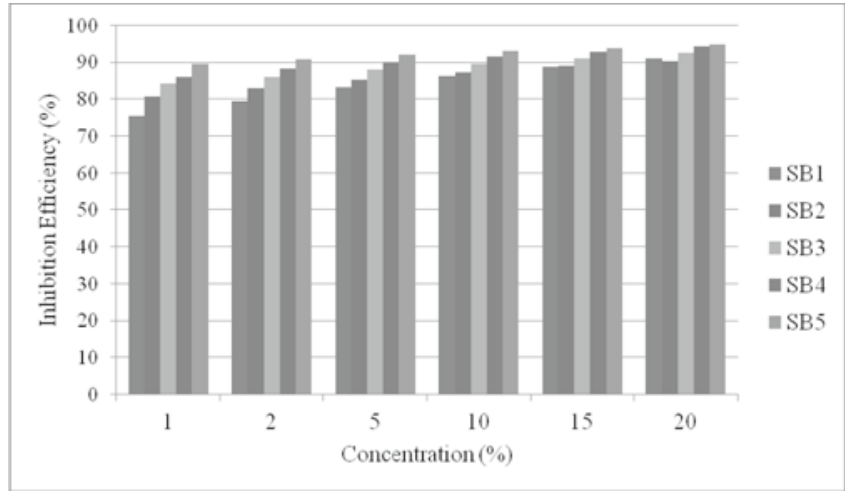

Figure 2. Inhibition efficiency v/s concentration of mild steel in $0.5 \mathrm{~N} \mathrm{HNO}_{3}$

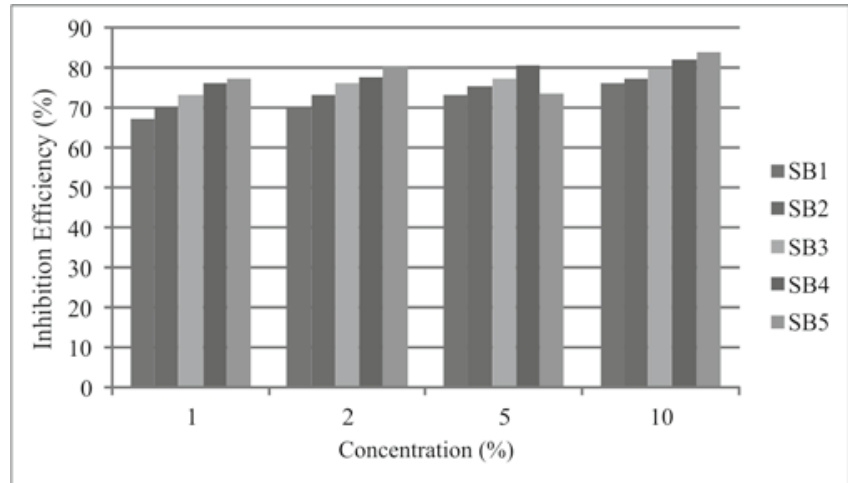

Figure 3. Inhibition efficiency $\mathrm{v} / \mathrm{s}$ concentration of mild steel in $0.1 \mathrm{~N} \mathrm{HNO}_{3}$ (Polarization) 
Table 1. Effect on Schiff bases on mass loss data for corrosion of mild steel in $0.1 \mathrm{~N}$ nitric acid. Effective area of specimen: $3.875 \mathrm{~cm}^{2}$. Temperature: $23 \pm 0.5^{\circ} \mathrm{C}$. Immersion Time: 72 hours

\begin{tabular}{|c|c|c|c|c|c|}
\hline $\begin{array}{l}\text { Inhibitors } \\
\text { Concentration } \\
{[\mathrm{mM}]}\end{array}$ & Mass loss [mg] & $\begin{array}{c}\text { Inhibition } \\
\text { Efficiency } \\
{[\eta \%]}\end{array}$ & $\begin{array}{l}\text { Corrosion rate } \\
{[\mathrm{mmpy}]}\end{array}$ & $\begin{array}{c}\text { Surface } \\
\text { Coverage }[\theta]\end{array}$ & $\log [\theta / 1-\theta]$ \\
\hline Blank & 1570.25 & & 381.88 & & \\
\hline \multicolumn{6}{|l|}{ SB1 } \\
\hline 1 & 394.68 & 74.87 & 95.99 & 0.7487 & 0.4740 \\
\hline 2 & 333.61 & 78.75 & 81.13 & 0.7875 & 0.5690 \\
\hline 5 & 279.74 & 82.18 & 68.03 & 0.8218 & 0.6640 \\
\hline 10 & 238.94 & 84.78 & 58.11 & 0.8478 & 0.7460 \\
\hline 15 & 200.71 & 87.22 & 48.81 & 0.8722 & 0.8340 \\
\hline 20 & 169.92 & 89.18 & 41.32 & 0.8918 & 0.9160 \\
\hline \multicolumn{6}{|l|}{ SB2 } \\
\hline 1 & 329.40 & 79.02 & 80.11 & 0.7902 & 0.5760 \\
\hline 2 & 294.29 & 81.26 & 71.58 & 0.8126 & 0.6370 \\
\hline 5 & 255.23 & 83.75 & 62.07 & 0.8375 & 0.7120 \\
\hline 10 & 220.87 & 85.93 & 53.72 & 0.8593 & 0.7860 \\
\hline 15 & 190.46 & 87.87 & 46.32 & 0.8787 & 0.8600 \\
\hline 20 & 163.03 & 89.62 & 39.66 & 0.8962 & 0.9360 \\
\hline \multicolumn{6}{|l|}{ SB3 } \\
\hline 1 & 294.32 & 81.26 & 71.58 & 0.8126 & 0.6370 \\
\hline 2 & 252.29 & 83.93 & 61.36 & 0.8393 & 0.7180 \\
\hline 5 & 211.85 & 86.51 & 51.52 & 0.8651 & 0.8070 \\
\hline 10 & 175.58 & 88.82 & 42.70 & 0.8882 & 0.9000 \\
\hline 15 & 143.65 & 90.85 & 34.94 & 0.9085 & 0.9970 \\
\hline 20 & 119.30 & 92.40 & 29.01 & 0.9240 & 1.0850 \\
\hline \multicolumn{6}{|l|}{ SB4 } \\
\hline 1 & 232.93 & 85.17 & 56.65 & 0.8517 & 0.7590 \\
\hline 2 & 200.71 & 87.22 & 48.81 & 0.8722 & 0.8340 \\
\hline 5 & 169.92 & 89.18 & 41.32 & 0.8918 & 0.9160 \\
\hline 10 & 145.77 & 91.72 & 35.45 & 0.9172 & 0.9900 \\
\hline 15 & 124.48 & 92.07 & 30.27 & 0.9207 & 1.0650 \\
\hline 20 & 107.68 & 93.14 & 26.19 & 0.9314 & 1.1300 \\
\hline \multicolumn{6}{|l|}{ SB5 } \\
\hline 1 & 192.79 & 87.72 & 46.89 & 0.8772 & 0.8540 \\
\hline 2 & 172.37 & 89.02 & 41.92 & 0.8902 & 0.9090 \\
\hline 5 & 151.97 & 90.32 & 36.99 & 0.9032 & 0.9700 \\
\hline 10 & 133.75 & 91.48 & 32.53 & 0.9148 & 1.0310 \\
\hline 15 & 117.54 & 92.51 & 28.59 & 0.9251 & 1.0920 \\
\hline 20 & 103.15 & 93.43 & 25.09 & 0.9343 & 1.1530 \\
\hline
\end{tabular}

The following order of the inhibition efficiency has been observed for five Schiff bases for mild steel in nitric acid for mass loss and the potentiostatic method. SB1 < SB2 < SB3 < SB4 < SB5

However, the values of inhibition efficiency obtained from the corrosion current $\left(\mathrm{I}_{\text {corr }}\right)$ values are lower than those obtained from the mass loss data. Similar observations have been reported earlier Tables (3-4). The potentiostatic polarization results for mild steel in $0.1 \mathrm{~N}$

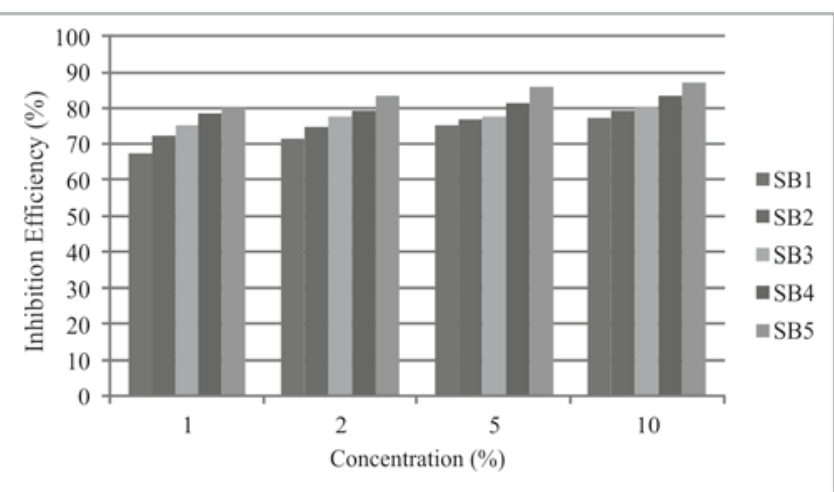

Figure 4. Inhibition efficiency v/s concentration of mild steel in $0.1 \mathrm{~N} \mathrm{HNO}_{3}$ (Polarization) nitric acid solution shows $83.97 \%$ inhibition efficiency while $0.5 \mathrm{~N}$ acid solution shows $87.32 \%$. The same results are presented in Figs. 3 and 4.

The order of the corrosion rate for mild steel in different test solutions observed by mass loss and potentiostate polarization method is in good agreement but the corrosion rate calculated from the potentiostatic method is higher than that of the mass loss method. It is expected that the corrosion rate is maximum in the beginning and decreases when equilibrium is attained. However, contrary to the earlier studies ${ }^{\mathbf{2 5 , 2 6}}$, the corrosion rates obtained from potentiostatic measurements are not significantly higher than those obtained by the mass loss methods.

The variation of the reaction number with the inhibitor concentration indicates that the reaction number decreases with increasing the inhibitor concentration (Table 5). The thermometric methods show the $96.78 \%$ inhibition efficiency for $1 \mathrm{~N}$ nitric acid solution while the $2 \mathrm{~N}$ nitric acid solution shows $97.98 \%$ whereas $4 \mathrm{~N}$ nitric acid solution shows $98.32 \%$ inhibition efficiency, respectively.

This process blocks active sites, hence decreases the corrosion rate. In the present study, it is assumed that 
Table 2. Effect on Schiff bases on mass loss data for corrosion of mild steel in $0.5 \mathrm{~N}$ nitric acid. Effective area of specimen: 3.875 $\mathrm{cm}^{2}$. Temperature: $23 \pm 0.5^{\circ} \mathrm{C}$. Immersion Time: 72 hours

\begin{tabular}{|c|c|c|c|c|c|}
\hline $\begin{array}{l}\text { Inhibitors } \\
\text { Concentration } \\
{[\mathrm{mM}]}\end{array}$ & Mass loss [mg] & $\begin{array}{c}\text { Inhibition } \\
\text { Efficiency } \\
{[\eta \%]}\end{array}$ & $\begin{array}{l}\text { Corrosion rate } \\
\text { [mmpy] }\end{array}$ & $\begin{array}{c}\text { Surface } \\
\text { Coverage }[\theta]\end{array}$ & $\log [\theta / 1-\theta]$ \\
\hline Blank & 3455.35 & & 840.34 & & \\
\hline \multicolumn{6}{|l|}{ SB1 } \\
\hline 1 & 844.77 & 75.55 & 205.45 & 0.7555 & 0.4900 \\
\hline 2 & 705.26 & 79.59 & 171.52 & 0.7959 & 0.5910 \\
\hline 5 & 574.75 & 83.37 & 139.78 & 0.8337 & 0.7000 \\
\hline 10 & 472.72 & 86.32 & 114.96 & 0.8632 & 0.8000 \\
\hline 15 & 386.36 & 88.82 & 93.96 & 0.8882 & 0.9000 \\
\hline 20 & 304.40 & 91.19 & 74.03 & 0.9119 & 1.0150 \\
\hline \multicolumn{6}{|l|}{ SB2 } \\
\hline 1 & 662.33 & 80.83 & 161.08 & 0.8083 & 0.6250 \\
\hline 2 & 283.64 & 83.11 & 141.94 & 0.8311 & 0.6920 \\
\hline 5 & 511.57 & 85.19 & 124.41 & 0.8519 & 0.7600 \\
\hline 10 & 436.37 & 87.37 & 106.13 & 0.8737 & 0.8400 \\
\hline 15 & 375.44 & 89.13 & 91.31 & 0.8913 & 0.9140 \\
\hline 20 & 335.81 & 90.28 & 81.67 & 0.9028 & 0.9680 \\
\hline \multicolumn{6}{|l|}{ SB3 } \\
\hline 1 & 539.26 & 84.39 & 131.15 & 0.8439 & 0.7330 \\
\hline 2 & 479.33 & 86.13 & 116.57 & 0.8613 & 0.7930 \\
\hline 5 & 413.22 & 88.04 & 100.49 & 0.8804 & 0.8670 \\
\hline 10 & 359.56 & 89.59 & 87.44 & 0.8959 & 0.9350 \\
\hline 15 & 308.25 & 91.08 & 74.97 & 0.9108 & 1.0090 \\
\hline 20 & 256.45 & 92.58 & 62.37 & 0.9258 & 1.0960 \\
\hline \multicolumn{6}{|l|}{ SB4 } \\
\hline 1 & 479.33 & 86.13 & 116.57 & 0.8613 & 0.7930 \\
\hline 2 & 401.63 & 88.38 & 97.88 & 0.8838 & 0.8810 \\
\hline 5 & 340.02 & 90.16 & 82.69 & 0.9016 & 0.9620 \\
\hline 10 & 291.24 & 91.57 & 70.83 & 0.9157 & 1.0360 \\
\hline 15 & 248.90 & 92.80 & 60.53 & 0.9280 & 1.1100 \\
\hline 20 & 206.41 & 94.30 & 50.20 & 0.9403 & 1.1970 \\
\hline \multicolumn{6}{|l|}{ SB5 } \\
\hline 1 & 364.79 & 89.44 & 88.525 & 0.8944 & 0.9280 \\
\hline 2 & 317.43 & 90.81 & 77.20 & 0.9081 & 0.9950 \\
\hline 5 & 276.84 & 91.99 & 67.33 & 0.9199 & 1.0600 \\
\hline 10 & 238.47 & 93.10 & 58.00 & 0.9310 & 1.1300 \\
\hline 15 & 209.57 & 93.94 & 50.97 & 0.9394 & 1.1900 \\
\hline 20 & 179.99 & 94.79 & 43.77 & 0.9479 & 1.2600 \\
\hline
\end{tabular}

the Schiff bases are adsorbed on the metal surface and decrease the surface area available for cathodic and anodic reaction to take place. Adsorption shows the collection of adsorb on the surface due to a cohesive force of attraction.

According to the Fontana ${ }^{27}$ for the more commonly used low carbon steel and steel alloys, $<1$ mpy is outstanding corrosion rate, 1-5 mpy is excellent, 5-20 mpy is good and can be used with caution, 20-50 is fair and can be used for good exposure, 50-200 mpy is poor and above 200 mpy is completely unacceptable.

But the corrosion rates are due to a significant contribution of nitrate salt available in water, hence the best promising results are obtained by decreasing the corrosion rate due to anisalidine derivatives.

\section{CONCLUSIONS}

1. Anislidine derivatives show a synergistic effect in controlling the corrosion of mild steel in acidic solution containing higher concentration of the inhibitor.

2. The protection film on the metal surface consists of mild steel-anisalidine derivatives complex and mild steel oxide.
3. Anisalidine derivatives form a complex on the anodic site of the metal surface through the oxygen atom and nitrogen atom of $-\mathrm{OH},-\mathrm{CHO},>\mathrm{C}=\mathrm{O}$, and $-\mathrm{NH}_{2}$ group.

\section{ACKNOWLEDGEMENT}

We are grateful to the department of Pure and Applied Chemistry, Maharshi Dayanand Sarswati University, (Ajmer) for the providing necessary facilities for the research work. 
Table 3. Potentiostatic polarization data for mild steel in $0.1 \mathrm{~N}$ nitric acid. Effective area of specimen: $3.875 \mathrm{~cm}^{2}$. Temperature: $23 \pm 0.5^{\circ} \mathrm{C}$

\begin{tabular}{|c|c|c|c|c|c|c|c|}
\hline $\begin{array}{l}\text { Inhibitor } \\
\text { Concentration } \\
\text { [mM] }\end{array}$ & $\begin{array}{l}\mathrm{OCP} \\
{[-\mathrm{mV}]}\end{array}$ & $\begin{array}{c}\text { Icorr } \\
\mathrm{mA} / \mathrm{cm}^{2}\end{array}$ & $\begin{array}{c}\text { Inhibition } \\
\text { Efficiency } \\
{[\eta \%]}\end{array}$ & $\begin{array}{c}\text { Corrosion } \\
\text { Rate }[\mathrm{mmpy}]\end{array}$ & $\begin{array}{c}\beta a \\
\mathrm{mV} / \text { decade }\end{array}$ & $\begin{array}{c}\beta c \\
-m V / d e c a d e\end{array}$ & $\begin{array}{c}\mathrm{Rp} \\
\text { [ohms] }\end{array}$ \\
\hline Blank & 583 & 912.65 & & 416.73 & 80 & 147 & 0.0246 \\
\hline \multicolumn{8}{|l|}{ SB1 } \\
\hline 1 & 575 & 298.25 & 67.32 & 136.19 & 85 & 149 & 0.0788 \\
\hline 2 & 574 & 271.51 & 70.25 & 123.98 & 86 & 149 & 0.0872 \\
\hline 5 & 573 & 244.43 & 73.25 & 111.47 & 87 & 150 & 0.0979 \\
\hline 10 & 572 & 218.12 & 76.10 & 99.60 & 89 & 152 & 0.1117 \\
\hline \multicolumn{8}{|l|}{ SB2 } \\
\hline 1 & 577 & 270.78 & 70.33 & 123.64 & 83 & 151 & 0.0859 \\
\hline 2 & 570 & 244.13 & 73.25 & 111.47 & 87 & 153 & 0.0986 \\
\hline 5 & 569 & 222.14 & 75.66 & 101.43 & 85 & 154 & 0.1071 \\
\hline 10 & 571 & 206.99 & 77.32 & 94.51 & 90 & 154 & 0.1192 \\
\hline \multicolumn{8}{|l|}{ SB3 } \\
\hline 1 & 569 & 243.40 & 73.33 & 101.14 & 87 & 152 & 0.0987 \\
\hline 2 & 569 & 217.03 & 76.22 & 99.10 & 87 & 155 & 0.1115 \\
\hline 5 & 568 & 206.99 & 77.32 & 94.51 & 90 & 153 & 0.1189 \\
\hline 10 & 560 & 182.44 & 80.01 & 83.30 & 92 & 154 & 0.1371 \\
\hline \multicolumn{8}{|l|}{ SB4 } \\
\hline 1 & 565 & 216.02 & 76.33 & 98.64 & 88 & 152 & 0.1120 \\
\hline 2 & 562 & 202.15 & 77.85 & 92.30 & 89 & 154 & 0.1212 \\
\hline 5 & 560 & 176.87 & 80.62 & 80.76 & 91 & 157 & 0.1414 \\
\hline 10 & 562 & 162.91 & 82.15 & 74.39 & 94 & 158 & 0.1571 \\
\hline \multicolumn{8}{|l|}{ SB5 } \\
\hline 1 & 562 & 206.72 & 77.35 & 94.39 & 90 & 150 & 0.1182 \\
\hline 2 & 564 & 180.52 & 80.43 & 82.43 & 90 & 153 & 0.1363 \\
\hline 5 & 560 & 161.08 & 73.55 & 73.55 & 93 & 155 & 0.1567 \\
\hline 10 & 561 & 140.09 & 83.97 & 72.97 & 95 & 159 & 0.1843 \\
\hline
\end{tabular}

Table 4. Potentiostatic polarization data for mild steel in $0.5 \mathrm{~N}$ nitric acid. Effective area of specimen: $3.875 \mathrm{~cm}^{2}$. Temperature: $23 \pm 0.5^{\circ} \mathrm{C}$.

\begin{tabular}{|c|c|c|c|c|c|c|c|}
\hline $\begin{array}{l}\text { Inhibitor } \\
\text { Concentration } \\
{[\mathrm{mM}]}\end{array}$ & $\begin{array}{c}\mathrm{OCP} \\
{[-\mathrm{mV}]}\end{array}$ & $\begin{array}{c}\text { Icorr } \\
\mathrm{mA} / \mathrm{cm}^{2}\end{array}$ & $\begin{array}{c}\text { Inhibition } \\
\text { Efficiency } \\
{[\eta \%]}\end{array}$ & $\begin{array}{c}\text { Corrosion } \\
\text { Rate [mmpy] }\end{array}$ & $\begin{array}{c}\beta a \\
\mathrm{mV} / \mathrm{dec} \text { ede }\end{array}$ & $\begin{array}{c}\beta c \\
-m V / d e c a d e\end{array}$ & $\begin{array}{c}\mathrm{Rp} \\
\text { [ohms] }\end{array}$ \\
\hline Blank & 584 & 1977.65 & & 903.01 & 82 & 148 & 0.0116 \\
\hline \multicolumn{8}{|l|}{ SB1 } \\
\hline 1 & 573 & 647.68 & 67.25 & 295.74 & 87 & 152 & 0.0371 \\
\hline 2 & 570 & 566.60 & 71.35 & 258.71 & 88 & 153 & 0.0428 \\
\hline 5 & 573 & 488.08 & 75.32 & 222.86 & 90 & 155 & 0.0507 \\
\hline 10 & 572 & 449.92 & 77.25 & 205.44 & 89 & 156 & 0.0547 \\
\hline \multicolumn{8}{|l|}{ SB2 } \\
\hline 1 & 569 & 546.82 & 72.35 & 249.68 & 86 & 152 & 0.0436 \\
\hline 2 & 568 & 494.61 & 74.99 & 225.84 & 89 & 154 & 0.0495 \\
\hline 5 & 567 & 457.23 & 76.88 & 208.78 & 91 & 157 & 0.0547 \\
\hline 10 & 565 & 408.98 & 79.32 & 186.74 & 92 & 159 & 0.0627 \\
\hline \multicolumn{8}{|l|}{ SB3 } \\
\hline 1 & 560 & 489.67 & 75.24 & 223.59 & 89 & 150 & 0.0495 \\
\hline 2 & 555 & 442.00 & 77.65 & 201.82 & 90 & 156 & 0.0561 \\
\hline 5 & 554 & 442.00 & 77.65 & 201.82 & 92 & 157 & 0.0570 \\
\hline 10 & 550 & 389.20 & 80.32 & 177.71 & 94 & 159 & 0.0659 \\
\hline \multicolumn{8}{|l|}{ SB4 } \\
\hline 1 & 559 & 422.23 & 78.65 & 192.79 & 91 & 151 & 0.0584 \\
\hline 2 & 555 & 408.78 & 79.33 & 186.65 & 93 & 158 & 0.0622 \\
\hline 5 & 550 & 370.81 & 81.25 & 169.32 & 94 & 159 & 0.0692 \\
\hline 10 & 547 & 329.67 & 83.33 & 150.53 & 95 & 157 & 0.0780 \\
\hline \multicolumn{8}{|l|}{ SB5 } \\
\hline 1 & 546 & 389.00 & 80.33 & 177.62 & 92 & 155 & 0.0644 \\
\hline 2 & 550 & 329.87 & 83.32 & 150.62 & 94 & 159 & 0.0778 \\
\hline 5 & 543 & 277.07 & 85.99 & 126.51 & 94 & 155 & 0.0917 \\
\hline 10 & 544 & 250.77 & 87.32 & 114.50 & 96 & 157 & 0.1032 \\
\hline
\end{tabular}


Table 5. Thermometric data for mild steel in $0.1 \mathrm{~N}, 2.0 \mathrm{~N}$ and $4.0 \mathrm{~N}$ nitric acid in presence of Schiff bases. Initial Temperature: $23 \pm 0.5^{\circ} \mathrm{C}$. Area of Specimen: $3.875 \mathrm{~cm}^{2}$

\begin{tabular}{|c|c|c|c|c|c|c|}
\hline \multirow{2}{*}{$\begin{array}{l}\text { Inhibitor } \\
\text { Concentration } \\
{[\mathrm{mM}]}\end{array}$} & \multicolumn{2}{|c|}{$1 \mathrm{~N}$} & \multicolumn{2}{|c|}{$2 \mathrm{~N}$} & \multicolumn{2}{|c|}{$4 \mathrm{~N}$} \\
\hline & $\mathrm{RN}$ & $\eta, \%$ & $\mathrm{RN}$ & $\eta, \%$ & $\mathrm{RN}$ & $\eta, \%$ \\
\hline Blank & 0.6830 & & 0.9450 & & 0.1710 & \\
\hline \multicolumn{7}{|l|}{ SB1 } \\
\hline 1 & 0.1427 & 79.10 & 0.1758 & 81.40 & 0.0270 & 84.20 \\
\hline 2 & 0.1205 & 82.35 & 0.1361 & 85.60 & 0.0230 & 86.53 \\
\hline 5 & 0.1003 & 85.32 & 0.1143 & 87.90 & 0.0168 & 90.20 \\
\hline 10 & 0.0818 & 88.02 & 0.1009 & 89.32 & 0.0120 & 93.00 \\
\hline 15 & 0.0669 & 90.21 & 0.0830 & 91.22 & 0.0101 & 94.10 \\
\hline 20 & 0.0522 & 92.35 & 0.0723 & 92.35 & 0.0074 & 95.70 \\
\hline \multicolumn{7}{|l|}{ SB2 } \\
\hline 1 & 0.1228 & 88.02 & 0.1503 & 84.10 & 0.0233 & 86.35 \\
\hline 2 & 0.1023 & 85.02 & 0.1162 & 87.70 & 0.0148 & 91.32 \\
\hline 5 & 0.0880 & 87.12 & 0.0936 & 90.10 & 0.0137 & 92.01 \\
\hline 10 & 0.0750 & 89.02 & 0.0699 & 92.60 & 0.0103 & 93.98 \\
\hline 15 & 0.0591 & 91.35 & 0.0491 & 94.80 & 0.0068 & 96.01 \\
\hline 20 & 0.0461 & 93.25 & 0.0463 & 95.10 & 0.0057 & 96.65 \\
\hline \multicolumn{7}{|l|}{ SB3 } \\
\hline 1 & 0.1048 & 84.65 & 0.1238 & 86.90 & 0.0199 & 88.35 \\
\hline 2 & 0.0871 & 87.25 & 0.0973 & 89.70 & 0.0148 & 91.35 \\
\hline 5 & 0.0727 & 89.35 & 0.0756 & 92.00 & 0.0119 & 93.03 \\
\hline 10 & 0.0591 & 91.35 & 0.0586 & 93.80 & 0.0097 & 94.32 \\
\hline 15 & 0.0522 & 92.35 & 0.0435 & 95.40 & 0.0082 & 95.21 \\
\hline 20 & 0.0402 & 94.12 & 0.0369 & 96.10 & 0.0057 & 96.65 \\
\hline \multicolumn{7}{|l|}{ SB4 } \\
\hline 1 & 0.0871 & 87.25 & 0.1030 & 89.10 & 0.0148 & 91.32 \\
\hline 2 & 0.0736 & 89.23 & 0.0728 & 92.30 & 0.0115 & 93.25 \\
\hline 5 & 0.0591 & 91.35 & 0.0558 & 94.10 & 0.0072 & 95.80 \\
\hline 10 & 0.0464 & 93.21 & 0.0506 & 94.65 & 0.0051 & 97.00 \\
\hline 15 & 0.0365 & 94.65 & 0.0389 & 95.88 & 0.0036 & 97.88 \\
\hline 20 & 0.0318 & 95.35 & 0.0312 & 96.70 & 0.0031 & 98.21 \\
\hline \multicolumn{7}{|l|}{ SB5 } \\
\hline 1 & 0.0682 & 90.02 & 0.0680 & 92.80 & 0.0131 & 92.32 \\
\hline 2 & 0.0591 & 91.34 & 0.0567 & 94.00 & 0.0097 & 94.32 \\
\hline 5 & 0.0463 & 93.22 & 0.0397 & 95.80 & 0.0062 & 96.35 \\
\hline 10 & 0.0395 & 94.22 & 0.0350 & 96.30 & 0.0055 & 96.78 \\
\hline 15 & 0.0318 & 95.35 & 0.0273 & 97.11 & 0.0032 & 98.10 \\
\hline 20 & 0.0220 & 96.78 & 0.0191 & 97.98 & 0.0029 & 98.32 \\
\hline
\end{tabular}

\section{LITERATURE CITED}

1. Nnabuk, O.E. (2010). Theoretical study on some amino acids and their potential activity as corrosion inhibitors for mild steel in $\mathrm{HCl}$. Molecular Simulation. 36 (5), 354-363. DOI: $10.1080 / 08927020903483270$.

2. Eddy, N.O., Ekwumemgbo, P.A., \& Mamza Paul, A.P. (2009). Ethanol extract of Terminalia catappa as a green inhibitor for the corrosion of mild steel in $\mathrm{H}_{2} \mathrm{SO}_{4}$. Green Chemistry Letters and Reviews 2 (4), 223-231. DOI: 10.1080/17518250903359941.

3. Bansiwal, A., Anthony, P. \& Mathur, S.P. (2000). Inhibitive effect of some Schiff bases on corrosion of aluminium in hydrochloric acid solutions. British Corrosion J. 35, 301-303. DOI: 10.1179/000705900101501380.

4. Sethi, T., Chaturvedi, A., Upadhyay, R.K. \& Mathur, S.P. (2008). Inhibition effect of nitrogen-containing ligands on corrosion of aluminium in acid media with and without $\mathrm{KCl}$, Polish J. Chem. 82 (3), 591-598. DOI: 4711, 35400017378176.0130.

5. Sethi, T., Chaturvedi, A. Upadhyay, R.K. \& Mathur, S.P. (2007). Corrosion inhibitory effects of some Schiff's bases on mild steel in acid media. J. Chil. Chem. Soc. 52, 1206-1213. DOI: $10.4067 /$ S0717-97072007000300003.

6. Sputnik, E. \& Ademoviv, Z. (1995). Proceeding of the $8^{\text {th }}$ European Symposium on corrosion inhibitors (8 SEIC) Ann. Univ. Ferrara, N.S. Sez. V, Suppl., 257.

7. Clubby, B.G. (1990). Chemical inhibitors for corrosion control, Royal Soc. Chem., Cambridge, pp141.
8. Gojic, M. \& Kosec, L. (1997). Embrittlement damage of low alloy Mn-V steel. ISIJ Int., 37 (7), 685-690. DOI: 0915-1559.

9. Nnabuk, O.E. Stevens, A.O. \& Ibiam, N.A. (2010). Ethanol extract of Ocimum gratissimum as a green corrosion inhibitor for the corrosion of mild steel in $\mathrm{H}_{2} \mathrm{SO}_{4}$. Green Chemistry Letters and Reviews, 1, 1-8. DOI: 10.1080/17518251003636428.

10. Govindaraju, K.M. Gopi, D. \& Kavitha, L. (2009). Inhibiting effects of 4-amino-antipyrine based schiff base derivatives on the corrosion of mild steel in hydrochloric acid. Journal of Applied Electrochemistry 39 (12), 2345-2352. DOI: 10.1007/ s10800-009-9920-4.

11. Behpour, M., Ghoreishi, S.M., Gandomi-Niasar, A., Soltani, N. \& Salavati-Niasari, M. (2009). The inhibition of mild steel corrosion in hydrochloric acid media by two Schiff base compounds. Journal of Materials Science 44 (10), 2444-2453, DOI: 10.1007/s10853-009-3309-y.

12. Nabel, A., Negm, F.M., Ghuiba, S.A., Mahmoud, S.M. \& Tawfik. (2011). Biocidal and anti-corrosive activities of benzoimidazol-3-ium cationic Schiff base surfactants. Engineering in Life Sciences 11 (5), 496-510. DOI: 10.1002/elsc.201000106.

13. Silva, A.B., Agostinho, S.M.L., Barcia, O.E., Cordeiro, G.G.O. \& D'Elia, E. (2006). The effect of cysteine on the corrosion of 304L stainless steel in sulphuric acid. Corrosion Science 48, 3668-3674. DOI: 10.1016/j.corsci.2006.02.003.

14. Putilova, I.N., Balizin, S.A. \& Baranmik, V.P. (1960). Metallic Corrosion Inhibitors, Pergmann Press, London.

15. Bereket, G., Yurt, A., Kandemir, S.U., Balaban, A. \& Erk, B. (2004). $5^{\text {th }}$ Advanced Batteries and Acccumulators-ABA. 
16. Kosari, A., Momeni, M., Parvizi, R., Zakeri, M., Moayed, M.H., Davoodi, A. \& Eshghi, H. (2011). Theoretical and electrochemical assessment of inhibitive behavior of some thiophenol derivatives on mild steel in $\mathrm{HCl}$. Corrosion Science. 53 (10), 3058-3067. DOI: 10.1016/j.corsci.2011.05.009.

17. Paul, O.A., Ladan, M. \& Takuma, S. (2012). Corrosion inhibition and adsorption behaviour for mild steel by Ficus glumosa gum in $\mathrm{H}_{2} \mathrm{SO}_{4}$ solution. African Journal of Pure and Applied Chemistry 6 (7), 100-106. DOI: 10.5897/AJPAC12.001.

18. Nnabuk Eddy, O. (2010), Fermentation product of Streptomyces griseus (albomycin) as a green inhibitor for the corrosion of zinc in $\mathrm{H}_{2} \mathrm{SO}$. Green Chemistry Letters and Reviews 3 (4), 307-314. DOI:10.1080/17518253.2010.486771.

19. Eddy, N.O. \& Odiongenyi, A.O. (2010). Corrosion inhibition and adsorption properties of ethanol extract of Heinsia crinata on mild steel in $\mathrm{H}_{2} \mathrm{SO}_{4}$, Pigment \& Resin Technology 39 (5), 288-295 DOI: 10.1108/03699421011076407.

20. Aiman Eid Al-Rawajfeh \& Ubeidulla Al-Qawabeha. (2007). Influence of diamond pressing process on corrosion resistance in steel. Materials Science and Engineering: A Volume 456 (1-2), 133-137. DOI: /10.1016/j.msea.2007.01.070.

21. Ashassi-Sorkhabi, H., Majidi, M.R. \& Seyyedi, K. (2004). Investigation of inhibition effect of some amino acids against steel corrosion in $\mathrm{HCl}$ solution. Applied Surface Science 225 (1-4), 176-185. DOI: 10.1016/j.apsusc.2003.10.007.

22. Arora, P., Kumar, S., Sharma, M.K. \& Mathur, S.P. (2007). Corrosion inhibition of aluminium by Capparis decidua in Acidic Media. E-J. Chem. 4, 450-456. DOI: 0973-4945; CODEN ECJHAO.

23. Ayşe Ongun Yüce \& Gülfeza Kardaş. (2012). Adsorption and inhibition effect of 2-thiohydantoin on mild steel corrosion in $0.1 \mathrm{M} \mathrm{HCl}$. Corrosion Science Volume 58, pp 86-94. DOI: 10.1016/j.corsci.2012.01.013.

24. Hoar, T.P. (1967). The production and breakdown of passivity of metals. Corrosion Science 7, pp 341-355.

25. Sharma, M.K., Arora, P., Kumar, S., Ratnani, R. \& Mathur, S.P. (2008). Inhibitive effect of Prosopis cineraria on mild steel in acidic media. Corrosion Engineering, Science and Technology 43 (3), 213-218. DOI: 10.1179/174327807X196816.

26. Saxena, N., Kumar, S. \& Mathur, S.P. (2009). Anisalidine derivative as corrosion inhibitor of mild steel in acidic media-I. Chemical Engineering Communication, 196, (12), 1451-1465. DOI: $10.1080 / 00986440902938881$.

27. Fontana, M.G. (1986). Corrosion Engineering (London: Mc Graw Hill publication) $3^{\text {rd }}$ Ed. pp. 172. 